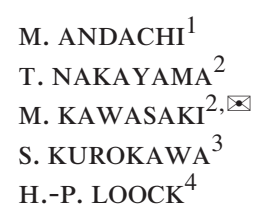

\section{Fiber-optic ring-down spectroscopy using a tunable picosecond gain-switched diode laser}

\author{
${ }^{1}$ Kyoto Prefectural Technology Center for Small and Medium Enterprises, Kyoto Research Park, \\ Kyoto 600-8813, Japan \\ 2 Department of Molecular Engineering, Kyoto University, Kyoto 615-8510, Japan \\ ${ }^{3}$ National Metrology Institute of Japan, National Institute of Advanced Industrial Science \\ and Technology, Tsukuba 305-8568, Japan \\ ${ }^{4}$ Department of Chemistry, Queen's University, Kingston, ON, Canada, K7L 3N6
}

\begin{abstract}
Received: 17 January 2007/Revised version: 21 February 2007 (C) Springer-Verlag 2007

ABSTRACT Visible and near-infrared laser light pulses were coupled into two different types of optical fiber cavities. One cavity consisted of a short strand of fiber waveguide that contained two identical fiber Bragg gratings. Another cavity was made using a loop of optical fiber. In either cavity $\sim 40 \mathrm{ps}$ laser pulses, which were generated using a custom-built gainswitched diode laser, circulated for a large number of round trips. The optical loss of either cavity was determined from the ring-down times. Cavity ring-down spectroscopy was performed on $200 \mathrm{pL}$ volumes of liquid samples that were injected into the cavities using a $100 \mu \mathrm{m}$ gap in the fiber loop. A detection limit of $20 \mathrm{ppm}$ of methylene blue dye in aqueous solution, corresponding to a minimum absorptivity of $\varepsilon C<6 \mathrm{~cm}^{-1}$, was realized.
\end{abstract}

PACS 42.62.Fi; 42.81.-i

\section{$1 \quad$ Introduction}

Cavity ring-down spectroscopy (CRDS) probes optical absorption of a species inside an optical cavity by measuring the change in the photon lifetime. This technique is an advancement over more conventional absorption methods because it achieves very long effective absorption paths and allows for absolute measurements of absorbance without the need for a reference path [1]. Originally, CRD was developed as a technique to greatly enhance the sensitivity and detection limit of gas-phase absorption spectroscopy, but there have also been recent attempts of adapting CRD spectroscopy to the condensed phase, either by inserting a flow cell in a conventional cavity [2,3] or by miniaturizing the cavity [4]. Alternatively, optical cavities consisting entirely of solid waveguide materials may be used to probe optical absorption of small volumes of liquid or of thin films - typically through interactions with the evanescent wave. Examples are quartz monoliths [5], microtoroidal resonators [6], fiber cavities [7-9] and fiber loops [10-12]. Some of these techniques were the subject of a recent review article [13].

Many commercial applications of CRD require inexpensive laser light sources that have a moderate wavelength

Fax: +81-75-383-2573, E-mail: kawasaki@moleng.kyoto-u.ac.jp tunability, but high repetition rates and short pulse widths. Therefore, it appears worthwhile to test the performance of gain-switched diode lasers in CRD applications. Gainswitching is a simple and effective technique of generating picosecond laser pulses from inexpensive commercial diode lasers $[14,15]$. Gain-switched diode lasers are commonly used in fluorescence lifetime measurements and a number of suppliers for fixed-wavelength laser systems exist. Generally, these gain-switched diode lasers are not suited for quantitative spectroscopic measurements since their short pulse width is frequently associated with low and strongly fluctuating output power. This makes conventional quantitative absorption spectroscopy or fluorescence spectroscopy difficult. On the other hand, cavity ring-down (CRD) spectroscopy is a technique that is largely insensitive to laser power fluctuations since the optical loss is obtained by measuring the rate of intensity loss instead of the absolute intensity [16-19].

As we will show, cavity ring-down measurements in a fiber cavity may be performed with light pulses that are generated by the gain-switching technique. Gain-switching can generate frequency-tunable sub-ns laser pulses from properly shaped electrical pulses into an inexpensive diode laser. Even if the laser intensity thus generated fluctuates, it does not affect the measurement of cavity ring-down decays. In this paper, we demonstrate that the gain-switching technique is useful in generating short and intense pulses that are suitable for ring-down measurements in two different types of fiber optic cavities: a cavity consisting of two identical fiber Bragg gratings (FBGs) and a cavity consisting of a fiber loop. We also demonstrate in a proof-of-principle absorption measurement that $20 \mathrm{ppm}$ of an organic dye solution in a $200 \mathrm{pL}$ volume can be detected, corresponding to a minimum detectable absorption loss of $(\varepsilon C)_{\min }<6 \mathrm{~cm}^{-1}$.

\section{$2 \quad$ Experimental \\ 2.1 Characterization of the laser light source}

A temperature tunable laser diode operating either at around $1563 \mathrm{~nm}$ or at $660 \mathrm{~nm}$ was used to obtain pulses by gain-switching. To achieve gain-switching operation, a part of sinusoidal wave of $100 \mathrm{MHz}$ (1/16 of a sine wave) was synthesized by a digital arbitrary waveform generator (Tektronix, AWG 710) and fed into a power amplifier (Kalmus, 704FC, maximum output $4 \mathrm{~W}$, gain $33 \mathrm{~dB}$ ). Through a power atten- 
uator $(3 \mathrm{~dB})$ for reduction of reflection intensity, the shaped current pulses were introduced into a DFB diode laser (Anritsu, $1563 \mathrm{~nm}$ ) or a single-transverse mode diode laser (Mitsubishi, $660 \mathrm{~nm}$ ) to generate pulses of $<40 \mathrm{ps}$ width (FWHM) at $1 \mathrm{MHz}$ repetition rate. The resulting temporal laser output profile of the DFB laser is shown in Fig. 1, together with the input electrical waveform.

The laser light was sent through an optical isolator before being amplified in two stages. The pulses were amplified first by $30 \mathrm{~dB}$ and then by $10-20 \mathrm{~dB}$ with two erbium- doped fiber amplifiers (IPG Laser GmbH, EAD10, EAD100). Analysis of a series of individual pulses showed that the shot-to-shot power fluctuations are less than $1 \%$ of the total intensity and the pulse width changes are below one picosecond. The ringdown waveforms were recorded using either a near-infrared GaAs detector (Agilent Technology, HP83482A: rise time $\sim 30 \mathrm{ps}$ ) or a photomultiplier tube (Hamamatsu Photonics, $\mathrm{R}-647$; rise time $<10 \mathrm{~ns})$. The output of the detectors was sampled and averaged by a $30 \mathrm{GHz}$ optical oscilloscope (Agilent Technology, HP83480A). When the repetition rate of the input current pulse was reduced from $1 \mathrm{MHz}$ to $100 \mathrm{kHz}$, the width of the laser output pulse remained at $<40 \mathrm{ps}$. Optical spectra of the diode laser pulses were measured with a spectrum analyzer (Ando Electronics, 6315B) and were found to be close to being transform limited. In order to reduce optical sideband intensity, a band pass filter $(0.3 \mathrm{~nm})$ was used before the light was introduced into the fiber cavity. Typical filtered emission spectra are shown in Fig. 2b for different temperatures of the laser diode. Compared to the continuous-wave (cw) operation, most of the spectral purity was retained.

(a)

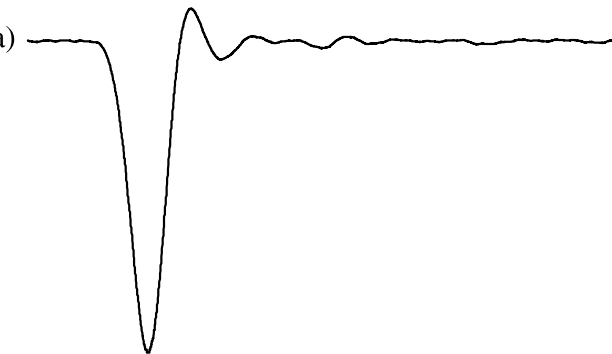

(c)

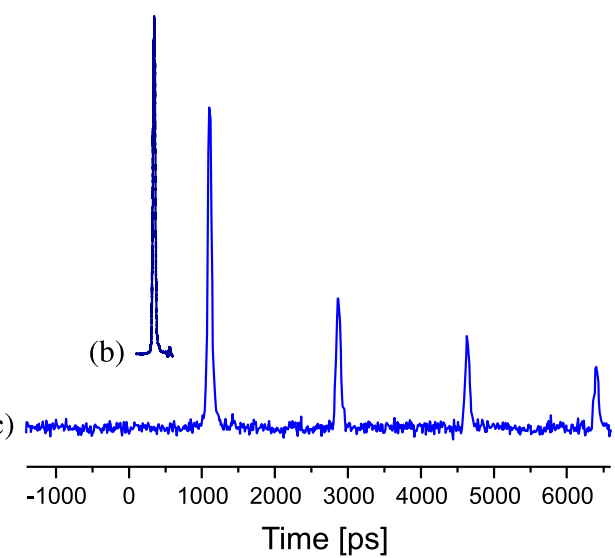

FIGURE 1 Time sequence showing (a) the electrical pulse and (b) the output laser pulse obtained with an oscilloscope (see also Fig. 2a) The ring-down waveform (c) was obtained from a $30.2 \mathrm{~cm}$ cavity consisting of two identical $99 \%$ reflectivity ( $20 \mathrm{~dB}$ ) fiber Bragg gratings (see also Fig. 4a)
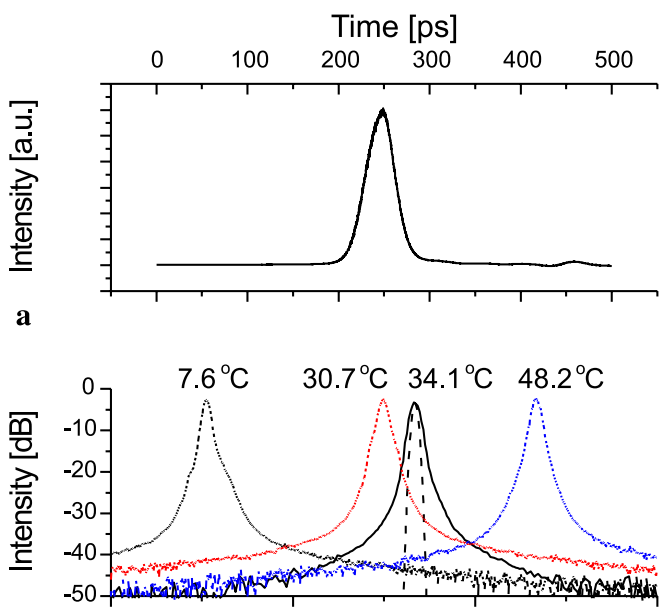

b

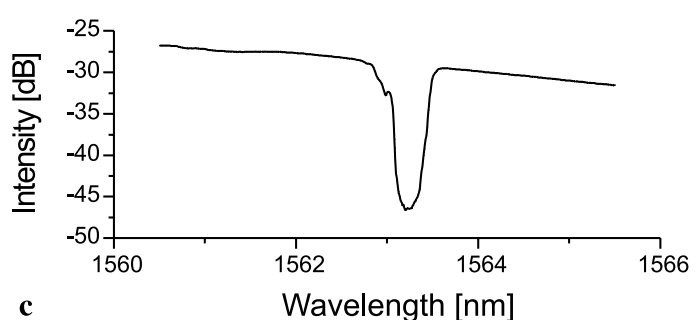

FIGURE 2 (a) Temporal laser profile showing a pulse width of $36 \mathrm{ps}$ and (b) emission spectra of the DFB laser at different temperatures. The expected spectrum for a transform limited pulse with a Gaussian temporal width of $36 \mathrm{ps}$ is superimposed as a dashed curve on the emission spectrum obtained at $34.1^{\circ} \mathrm{C}$. Panel (c) shows the attenuation spectrum of a single $20 \mathrm{~dB}$ FBG obtained using a cw broadband light source

\subsection{Waveguide cavities}

Two different types of cavities were used: a cavity based on two identical fiber Bragg gratings and a fiber loop cavity. For the second cavity type, a simple interface with an aqueous dye solution was used. The experimental configurations are schematically shown in Fig. 3.

\section{$2.3 \quad$ Fiber cavity $C R D$}

FBGs with reflectivities from $R=0.90(10 \mathrm{~dB})$ to $R=0.9999(40 \mathrm{~dB})$ at around $1563 \mathrm{~nm}$ were fabricated by Tatsuta Electric Wire and Cable Co. at the two ends of a single-mode fiber strand (30.2 cm length). Attenuation spectra of the FBGs were measured using the erbium-doped fiber amplifier (IPG Laser GmbH, EAD100) as a cw broadband light source. An example of the attenuated spectrum through a single FBG $(R=0.99)$ is shown in Fig. 2c. Ringdown measurements were made by introducing the amplified diode laser pulses into the fiber cavity through one of the FBGs. A typical temporal decay of pulse trains truncated at $200 \mathrm{~ns}-$ is shown in Fig. 4. This decay corresponded to an effective $R=0.996$. When $R=0.9999$ the absolute signal intensity from the exit FBG was too weak to be detected. Typically 500-5000 laser shots were averaged. Despite the high sampling rate of $20 \mathrm{GS} / \mathrm{s}$, the inset in Fig. 4a shows that the uncertainties in the ring-down measurement could have been reduced by an even higher sampling rate. 


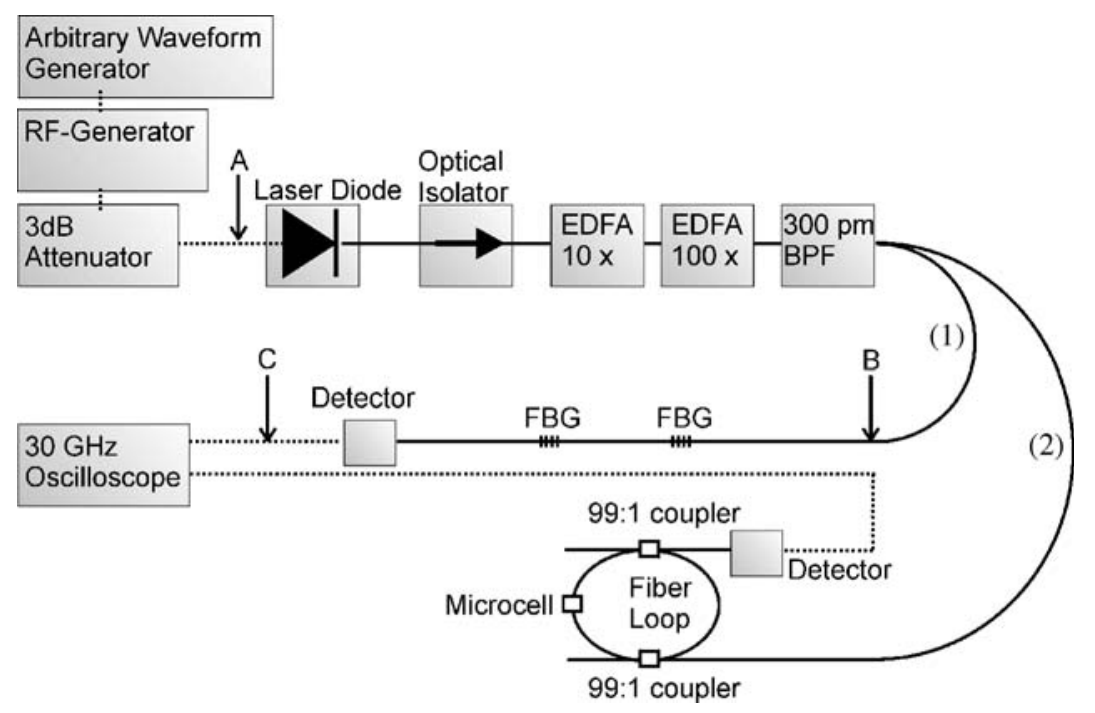

FIGURE 3 Scheme of the experimental setup. The configurations are shown for (1) the cavity defined by two FBGs and (2) for the $4.69 \mathrm{~m}$ fiber loop. The letters (A-C) indicate points at which the temporal profile of the electrical or optical pulses was measured (see Fig. 1). The spectrum shown in Fig. $2 \mathrm{~b}$ was obtained at measurement point "B"

The fiber loop CRD configuration used a loop of multimode fiber (125/50, Sumitomo Electric Industries) $4.69 \mathrm{~m}$ in length. The loop contained two identical fiber optic couplers with a 99: 1 split ratio (Tatsuta Electric Wire and Cable Co.) by which light from the laser was introduced into the loop and coupled out to the photomultiplier detector, respectively. The split ratio was chosen to provide directional input/output coupling of the radiation and to give reasonably low optical losses.

In a separate experiment a similar loop was used, but a small gap in the loop allowed for the introduction of liquid between the fiber ends. A microcell of about $100 \mu \mathrm{m}$ length and $50 \mu \mathrm{m}$ diameter (Moritex Co.) was specified to have a $0.43 \mathrm{~dB}$ loss at $1550 \mathrm{~nm}$.

\section{$3 \quad$ Results \\ 3.1 FBG cavity}

Figure 4 displays a typical ring-down trace obtained with two identical $30 \mathrm{~dB}$ FBGs. To obtain the trace, 4096 laser pulses have been averaged. We observed a biexponential decay in which the fast decay process is due to light that is only poorly trapped by the FBG cavity. It is plausible that this corresponds to modes at the laser wavelength that are guided largely by the cladding, but because of the different effective refractive index of these cladding modes, we expect those not to be retained by the FBG cavity. Alternatively, the fast decay process may be broadband amplified spontaneous emission (ASE), which is typically emitted by diode laser sources. In our case, ASE has been reduced greatly by the bandpass filter, as was verified using a spectrum analyzer, but even a contribution of $10^{-6}(60 \mathrm{~dB})$ would be comparable to the laser intensity behind a cavity consisting of two $30 \mathrm{~dB}$ FBGs. This was discussed by Naus et al. for the case of a "white" cavity with a pseudo-continuous mode structure [20]. In any case, these modes give rise to intense peaks for the first few roundtrips in the ring-down transients. In the determination of the ring-down time, the first four roundtrips were, therefore, excluded from the exponential fit.
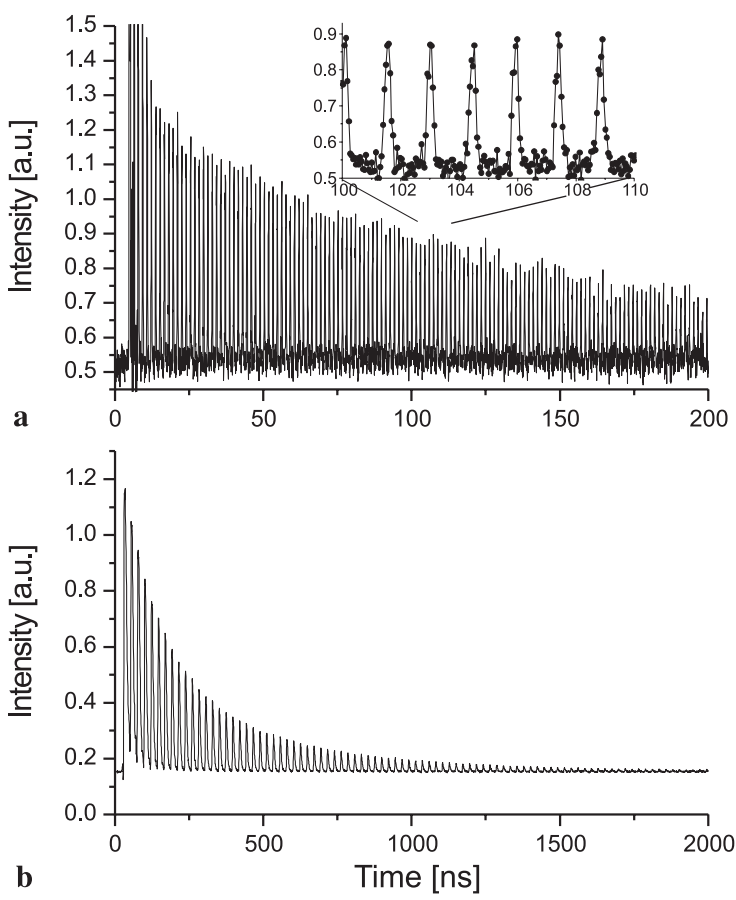

FIGURE 4 (a) Ring-down waveform from a cavity containing two identical $30 \mathrm{~dB}$ FBGs and excited by a 40 ps pulse of $1563 \mathrm{nmlight}$. The ring-down time is obtained from an exponential fit to the peak amplitudes as $\tau=114 \mathrm{~ns}$. (b) Ring-down signal obtained using a $4.69 \mathrm{~m}$ fiber loop cavity excited with a pulse of $660 \mathrm{~nm}$ light. A biexponential fit to the peak amplitudes gives ring-down times of $399 \mathrm{~ns}$ and $118 \mathrm{~ns}$

The envelope of the waveform was fit to an exponential decay with a ring-down time of $\tau=114 \pm 8 \mathrm{~ns}$. The loss per pass can be calculated from the roundtrip-time (here $t_{\mathrm{R}}=1.46 \pm$ $0.03 \mathrm{~ns}$ ) and the ring-down time, $\tau$, using

$\tau=\frac{t_{\mathrm{R}}}{2(-\ln R+\alpha L)}$.

Here, $R$ is the transmission through the splice between the FBGs and $\alpha L$ is the absorption loss due to the fiber material of length $L=30.2 \pm 0.7 \mathrm{~cm}$. The latter term can be neglected since it is specified as $<3 \mathrm{~dB} / \mathrm{km}$ or less than $1 \times 10^{-3} \mathrm{~dB} / 30 \mathrm{~cm}$. The term in brackets corresponds to 
a loss of $99.36 \%(0.027 \mathrm{~dB})$ - a value that is consistent with the average loss associated with the nominally $>99.9 \%$ reflective FBGs.

\subsection{Fiber loop cavity ring-down measurements}

To adapt the conventional decay formalism to the case of a uni-directional fiber loop cavity containing a microcell, (1) is changed to include the intrinsic losses of the system as well as a term for the loss due to external absorption, $\varepsilon C d$. The factor of two in the denominator of (1) is not needed since the sample absorbs light only once per roundtrip [11]

$$
\tau=\frac{t_{\mathrm{R}}}{-\ln T+\alpha L+\varepsilon C d} \text {. }
$$

Figure $4 \mathrm{~b}$ shows the decay of an optical pulse injected into a closed fiber loop, i.e., here $\varepsilon C d=0$. Here, 4096 laser pulses have been averaged and the peak amplitudes were fitted to a biexponential decay with ring-down times of $118 \pm$ $3 \mathrm{~ns}$ and $399 \pm 11 \mathrm{~ns}$, respectively. The biexponential nature of the ring-down envelope indicates that cladding and core modes decay at different rates [11]. Their loss per roundtrip is $0.84 \mathrm{~dB}$ and $0.25 \mathrm{~dB}$, respectively. From the manufacturers specifications, the multimode fiber material's attenuation is $<10 \mathrm{~dB} / \mathrm{km}$ and losses of the slower decaying core modes are calculated as $<0.05 \mathrm{~dB}$ per roundtrip due to absorption and scattering in the fiber optic cable $(L=4.69 \mathrm{~m})$. Similarly, a loss of about $0.1 \mathrm{~dB}$ for each of the fiber-fiber couplers is consistent with the specifications of the fiber-fiber couplers, i.e., a split ratio of $99: 1$ (corresponding to a loss of $0.044 \mathrm{~dB}$ ) and an insertion loss of about $0.05 \mathrm{~dB}$. No specifications for waveguide absorption or coupler losses are available for the cladding modes, but we note that in work done previously using a Nd:YAG pumped dye laser system, optical losses were determined as about $40 \mathrm{~dB} / \mathrm{km}$ at $810 \mathrm{~nm}$ in similar waveguide material $[11,21]$. Extrapolating to $660 \mathrm{~nm}$ we can estimate losses of about $0.5 \mathrm{~dB}$ per roundtrip $(100 \mathrm{~dB} / \mathrm{km})$ in the cladding and correspondingly higher insertion losses of about $0.2 \mathrm{~dB}$ at each of the two couplers. These estimated numbers are again consistent with a total loss of $0.84 \mathrm{~dB}$ per roundtrip.

To test the capabilities of the fiber ring-down technique for measuring absorption in a microcell that was filled with an aqueous solution of the dye methylene blue, the ring-down time at $660 \mathrm{~nm}$ was measured as a function of dye concentration (Figs. 5 and 6). Considering the uncertainties in the measurements, it can be seen from the graph that a $20 \mathrm{ppm}$ $(50 \mu \mathrm{M})$ sample can readily be detected, giving an absolute detection limit for 10 femtomoles of methylene blue. The same result is obtained using [22]

$(\varepsilon C)_{\min }=\frac{L}{c d \tau_{0}} \frac{\Delta \tau_{\min }}{\tau_{0}}$,

with the speed of light $c=c_{0} / 1.46, d=100 \mu \mathrm{m}, L=4.69 \mathrm{~m}$ and $\tau_{0}=45 \mathrm{~ns}$. The absorption coefficient is given with respect to base $e$ as $\varepsilon=1.5 \times 10^{5} \mathrm{~L} \mathrm{~mol}^{-1} \mathrm{~cm}^{-1}$, which was measured by a spectrophotometer (Shimazu, UV-1600) using a $1 \mathrm{~cm}$ cell. Given that a minimum change in $\Delta \tau_{\min }=5 \mathrm{~ns}$ can be measured, the detection limit for our $200 \mathrm{pL}$ "absorption cell" can be placed at an absorptivity of $(\varepsilon C)_{\min }<6 \mathrm{~cm}^{-1}$ and a detection limit of $<50 \mu \mathrm{M}$ is obtained. When using a de-

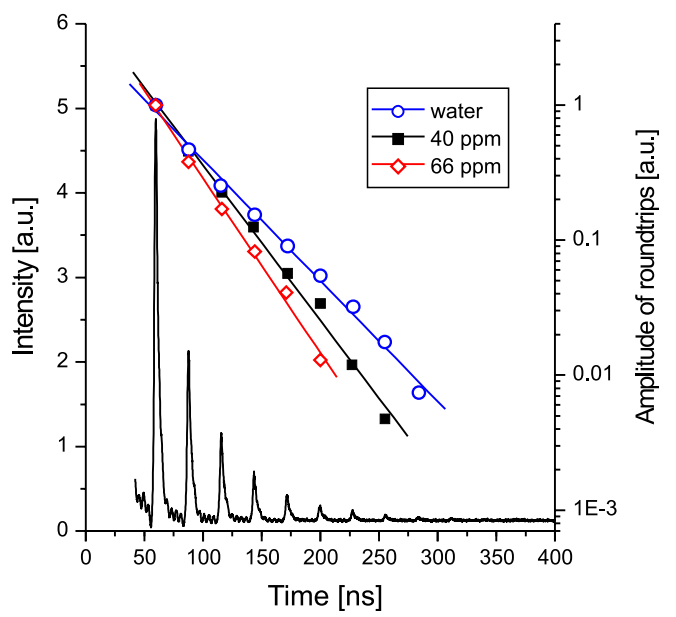

FIGURE 5 Exponential decay of the roundtrip intensity shown for the $40 \mathrm{ppm}$ solution of methylene blue, which was introduced into a gap in the fiber loop. On a logarithmic scale the exponential decay of the peak amplitudes for distilled water (circles) $40 \mathrm{ppm}$ (squares) and $66 \mathrm{ppm}$ (diamonds) is shown

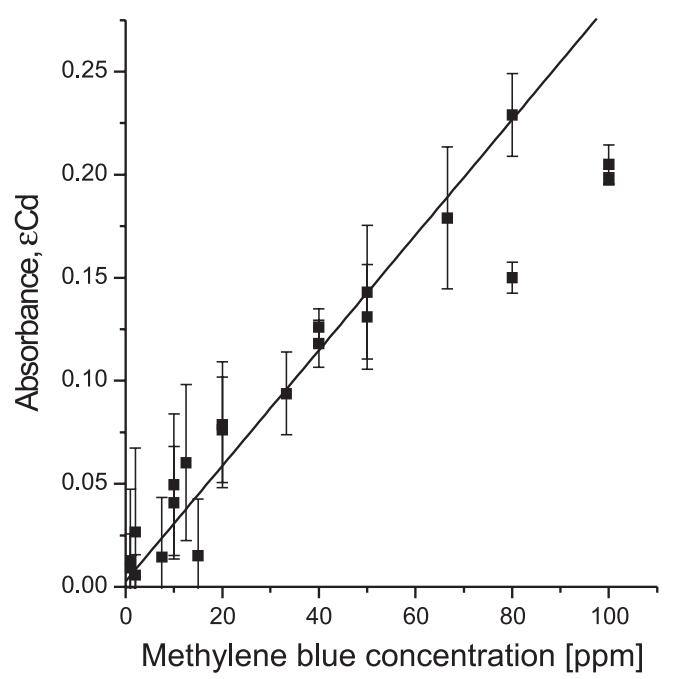

FIGURE 6 Absorbance, $\varepsilon C d$, measured using a $100 \mu \mathrm{m}$ cell in a fiber loop that was filled with an aqueous solution of methylene blue. Absorbance measurements at the two highest concentrations were omitted from the linear fit

tection system with a temporal response that is matched to the laser pulse width, a minimal detectable absorption loss of $(\varepsilon C)_{\min }<0.04 \mathrm{~cm}^{-1}$ is feasible with this device.

4

\section{Discussion}

It is necessary to highlight cases in which the use of gain-switched diode lasers in CRD is advantageous over other light sources. Using narrow band $(\sim 1 \mathrm{MHz}) \mathrm{cw}$ lasers clearly allows for superior sensitivity, spectral resolution and detection limit compared to pulsed lasers. This is true provided that they are coupled into cavities in which a single cavity mode may be excited. In this case, the laser bandwidth is small compared to the free spectral range of the cavity, and build-up of the cavity intensity can be achieved by accidental or intentional tuning of the cavity length or the laser wavelength. Once the cavity intensity exceeds a given threshold, the laser is quickly turned off and the ring-down event is monitored. 
Active and resonant locking of the laser line to the cavity may provide additional sensitivity. In their report, Mazurenka et al. have reviewed many of these experimental schemes [22]. Of course, active or passive mode-locking of cw-lasers becomes impossible once the free spectral range of the cavity is smaller than the linewidth of even a cw-laser. These limitations apply to cavities that consist of, e.g., very long single-mode fiber loops or multimode fiber loops such as those presented in this article. In these cases, using picosecond pulsed lasers have an advantage over switched cw-lasers.

As was pointed out in a landmark paper by Romanini and Lehmann, only light resonant with the longitudinal modes of the cavity can be coupled into the cavity and subsequently ring-down - regardless of whether the laser source is pulsed or continuous [18]. Most pulsed lasers, including pulsed diode lasers, have a bandwidth $(\mathrm{GHz})$ that is larger than the typical free-spectral range of the cavity (about $150 \mathrm{MHz}$ for a $1 \mathrm{~m}$ cavity), so that more than one longitudinal cavity mode is excited at a time [22]. In addition, a number of transverse modes may also be excited. In the study presented here, a single mode fiber is used in conjunction with a short cavity $(L=30.2 \mathrm{~cm})$ and a narrow line width laser could have been used to excite a single cavity mode. However, the linewidth of the diode laser $(150 \mathrm{pm}, \sim 3.7 \mathrm{GHz})$ is considerably broader than the free spectral range of the FBG cavity $(500 \mathrm{MHz})$, so that a few cavity modes are always simultaneously excited.

In the case of such a "pseudo-continuous" mode structure, fast intensity modulation of a cw-laser and measurement of the phase difference between the light entering and exiting the cavity presents an alternative way to use inexpensive cw-lasers [23, 24]. One example of such a cavity is the multimode fiber loop that was recently used in applications of phase shift CRD for absorption measurements in very small liquid samples $[25,26]$. The main advantage of phase shift CRD over time-resolved CRD lies in its ability to resolve ring-down times with an accuracy better than $1 \%$, regardless of the absolute value of the ring-down time. Therefore, sub-nanosecond time resolution can readily be obtained using laser diodes that are similar to those described here simply by increasing the modulation frequency of the laser light. However, compared to time-resolved measurements, multi-exponential decays may lead to difficulties in the analysis if not properly taken into account $[27,28]$.

Amplified gain-switched diode lasers with pulsewidths of less than $100 \mathrm{ps}$ are commercially available. Their fixed emission wavelengths span the entire spectrum from about $375 \mathrm{~nm}$ to $>1600 \mathrm{~nm}$. Commercial gain-switched diode lasers are mostly used for fluorescence lifetime measurements, and would be well suited for fixed wavelength CRD experiments. In principle, their short pulse widths permit measurements of ring-down times with sub-nanosecond accuracy - a feat that would otherwise only be possible by substantially more expensive laser systems. In both configurations presented here, the time resolution was limited to about $\Delta \tau_{\min }=1-5 \mathrm{~ns}$ by the response time of the detectors, but not by the lasers.

The potential uses for gain-switched lasers in analytical CRD applications were considerably extended by observing that the lasers were temperature tunable without loss in spectral purity and pulse width. While the tuning range is limited to a few nanometers, this may be sufficient for many analyti- cal applications such as gas phase isotope ratio measurements and quantification through individual rotational lines.

We note that the data-acquisition rate for the presented gain-switched laser system is comparably low. This is due to the low duty cycle and the large number of laser shots needed to acquire an averaged waveform on the optical oscilloscope. For time-resolved absorption measurements in microfluidic devices or capillaries, the technique would have to be combined with a much faster data acquisition system.

In summary, we conclude that wavelength tunable gainswitched laser diodes are an inexpensive and efficient means to generate picosecond laser pulses and that their spectral characteristics are well suited for CRD spectroscopy. While this was demonstrated for the specific case of optical waveguide cavities, their application range can readily be extended to include "conventional" CRD cavities, which are designed for gas-phase spectroscopy applications.

ACKNOWLEDGEMENTS HPL acknowledges the Japan Society for Promotion of Sciences for a visiting fellowship. MK thanks the Optoelectronic Industry and Technology Development Association for financial support.

\section{REFERENCES}

1 M.D. Wheeler, S.M. Newman, A.J. Orr-Ewing, M.N.R. Ashfold, J. Chem. Soc. Faraday Trans. 94, 337 (1998)

2 K.L. Snyder, R.N. Zare, Anal. Chem. 75, 3086 (2003)

3 K.L. Bechtel, R.N. Zare, A.A. Kachanov, S.S. Sanders, B.A. Paldus, Anal. Chem. 77, 1177 (2005)

4 B. Bahnev, L. van der Sneppen, A.E. Wiskerke, F. Ariese, C. Gooijer, W. Ubachs, Anal. Chem. 77, 1188 (2005)

5 A.C.R. Pipino, J.W. Hudgens, R.E. Huie, Rev. Sci. Instrum. 68, 2978 (1997)

6 D.K. Armani, T.J. Kippenberg, S.M. Spillane, K.J. Vahala, Nature 421, 925 (2003)

7 T. von Lerber, M.W. Sigrist, Appl. Opt. 41, 3567 (2002)

8 D.E. Vogler, M.G. Muller, M.W. Sigrist, Appl. Opt. 42, 5413 (2003)

9 M. Gupta, H. Jiao, A. O'Keefe, Opt. Lett. 27, 1878 (2002)

10 G. Stewart, K. Atherton, H.B. Yu, B. Culshaw, Meas. Sci. Technol. 12, 843 (2001)

11 R.S. Brown, I. Kozin, Z. Tong, R.D. Oleschuk, H.P. Loock, J. Chem. Phys. 117, 10444 (2002)

12 P.B. Tarsa, P. Rabinowitz, K.K. Lehmann, Chem. Phys. Lett. 383, 297 (2004)

13 C. Vallance, New J. Chem. 29, 867 (2005)

14 H. Ito, H. Yokohama, S. Murata, H. Inaba, Electron. Lett. 15, 738 (1979)

15 C. Lin, P. Liu, T. Damen, D. Eilenberger, R. Hartman, Electron. Lett. 16, $600(1980)$

16 A. O'Keefe, D. Deacon, Rev. Sci. Instrum. 59, 2544 (1988)

17 J.J. Scherer, J.B.P. O'Keefe, R.J. Saykally, Chem. Rev. 97, 25 (1997)

18 K.K. Lehmann, D. Romanini, J. Chem. Phys. 105, 10263 (1996)

19 K. Busch, M. Busch, Cavity-Ringdown Spectroscopy: An UltratraceAbsorption Measurement Technique, ed. by K. Busch, M. Busch (American Chemical Society, Washington, DC, 1999)

20 H. Naus, I.H.M. van Stokkum, W. Hogervorst, W. Ubachs, Appl. Opt. 40, $4416(2001)$

21 Z.G. Tong, M. Jakubinek, A. Wright, A. Gillies, H.P. Loock, Rev. Sci. Instrum. 74, 4818 (2003)

22 M. Mazurenka, A.J. Orr-Ewing, R. Peverall, G.A.D. Ritchie, Ann. Rep. Prog. Chem. C 101, 100 (2005)

23 R. Engeln, G. von Helden, G. Berden, G. Meijer, Chem. Phys. Lett. 262, 105 (1996)

24 Z. Tong, A. Wright, T. McCormick, H.-P. Loock, Anal. Chem. 76, 6594 (2004)

25 H.-P. Loock, TrAC-Tr Anal. Chem. 25, 655 (2006)

26 R.K. Li, H.P. Loock, R.D. Oleschuk, Anal. Chem. 78, 5685 (2006)

27 J. van Helden, D.C. Schram, R. Engeln, Chem. Phys. Lett. 400, 320 (2004)

28 V.L. Kasyutich, P.A. Martin, R.J. Holdsworth, Chem. Phys. Lett. 430, $429(2006)$ 